\title{
Ebola virus disease: assessment of knowledge, attitude and practice of nursing students of a Nigerian University
}

\author{
Aniekan J Etokidem ${ }^{1,2}$, Boniface U Ago ${ }^{3,4}$, Mary Mgbekem ${ }^{5}$, Affiong Etim ${ }^{6}$, Eno Usoroh ${ }^{7}$, Anastasia Isika ${ }^{1,2}$
}

1. Department of Community Medicine, University of Calabar, Calabar, Nigeria.

2. Department of Community Medicine, University of Calabar Teaching Hospital, Calabar, Nigeria.

3. Department of Obstetrics and Gynaecology, University of Calabar, Calabar, Nigeria.

4. Department of Obstetrics and Gynaecology, University of Calabar Teaching Hospital, Calabar, Nigeria.

5. Department of Nursing Science, University of Calabar, Calabar, Nigeria.

6. Department of Physiology, University of Calabar, Calabar, Nigeria.

7. KNCV-Nigeria/Challenge'TB, Akwa Ibom State, Nigeria.

\begin{abstract}
Background: Ebola virus disease has a high case fatality rate. Health care providers have a key role to play in its management and prevention.

Objectives: The aim of this study was to determine the knowledge, attitude and practice regarding Ebola virus disease (EVD) among students of Nursing in the University of Calabar, Calabar, Nigeria.

Methods: This was a descriptive cross-sectional study. A semi-structured,self-administered questionnaire was administered to 178 nursing students. The respondents were selected using systematic random sampling.

Results: Lecturers were the commonest source of information regarding EVD as reported by $84.5 \%$ of the respondents followed by television $(76.3 \%)$. Nearly $27 \%$ of respondents indicated that the fear of EVD had dampened their interest and enthusiasm in the nursing profession. Nearly $12 \%$ indicated that they would not take part as theatre nurses in the surgical operation of a patient who had been certified cured of EVD. A similar proportion indicated their unwillingness to attend to a woman in labour even if she had been certified cured of EVD.

Conclusion: Majority of the students were knowledgeable about EVD. Although majority of them had favorable attitude towards EVD patients, there was evidence of stigmatizing and discriminatory attitude that needs to be addressed.
\end{abstract}

Keywords: Ebola, infection, virus, disease, haemorrhagic, fever, Nigeria.

DOI: https://dx.doi.org/10.4314/ahs.v18i1.9

Cite as: Etokidem AJ, Ago BU, Mgbekem M, Etim A, Usorob E, Isika A. Ebola virus disease: assessment of knowledge, attitude and practice of nursing students of a Nigerian University. Afri Health Sci 2018;18(1): 55-65. https:// dx.doi.org/10.4314/abs.v18i1.9

\section{Introduction}

Ebola virus disease (EVD), which was formerly known as Ebola haemorrhagic fever (EHF), first appeared in 1976
Corresponding author:
Aniekan Jumbo Etokidem,
Department of Community Medicine,
Faculty of Medicine, University of Calabar
Calabar, Nigeria
Phone: +2348038227878
Email: etokidem@etokidem.com

in 2 simultaneous outbreaks; one in present day Nzara in South Sudan and the other in Yambuku, Democratic Republic of Congo. The latter occurred in a village near the Ebola River, after which the disease was named. ${ }^{1,2}$ Between 1976 and 2013, up to 20 outbreaks had been reported, resulting in about 2500 cases in 6 countries namely, the Democratic Republic of Congo, Sudan, Gabon, Côte d'Ivoire, Uganda and the Republic of the Congo. ${ }^{3}$ EVD is caused by a virus of the family Filoviridae, genus Ebola virus. The genus Ebola virus is divided into five different species namely Zaire, Sudan, TaiForest, Bundibugyo, and Reston viruses. The virus is highly virulent in humans 
although each genus has varying degree of virulence. Fruit bats and non-human primates constitute the natural reservoir of the virus. Transmission of the virus is through contact with blood, body secretions and fluids such as milk, semen as well as tissues of infected persons either while they are alive or immediately after their death. The disease has an incubation period of 2 to 21 days. ${ }^{4}$

The case fatality rate could be as high as $90 \%$, especially for the Zaire Ebola virus which is the most fatal., ${ }^{5,6}$ The symptoms of the disease include fever, asthenia, diarrhoea, headache, vomiting, abdominal pain, sore throat, joint aches, conjunctivitis muscle aches and chest pain. ${ }^{5,6,7,8}$

West Africa experienced an outbreak of EVD in January 2014 starting in Guinea and spreading to Sierra Leone and Liberia. Within months, it had spread to Mali, Senegal and Nigeria. By the end of December, 2014, there were 20,200 confirmed cases of EVD reported with Guinea reporting 2707 cases and 1708 deaths, giving a case fatality rate of $63.1 \%$. Liberia had reported 8018 cases with 3423 deaths, giving a case-fatality rate of $42.7 \%$. Sierra Leone had reported 9446 cases with 2758 deaths, giving a case-fatality rate of $29.2 \%$. Three West African countries that also reported confirmed cases include Nigeria (20 cases with 8 deaths, case fatality rate $40 \%$ ), Mali ( 8 cases with 6 deaths, case-fatality rate 75\%) and Senegal (1 case, no death, case fatality zero). ${ }^{9}$

The first case of EVD was introduced into Nigeria on $20^{\text {th }}$ July 2014 when the diagnosis was made in a Lagos hospital in a Liberian diplomat whose journey to attend a conference in Calabar, Cross River State of Nigeria, was aborted due to the illness and his subsequent death. Subsequently, a chain of transmission was set off that infected a total of 20 people, 8 of whom died of the infection. ${ }^{9,10}$

Health care providers, by virtue of the likely exposure to the blood and body fluids of their patients in the course of providing care, are at risk of contracting the disease from their clients, especially if they do not observe standard precautions and use personal protective equipment.

Study among students in different parts of the world have shown interesting results. A study in Malaysia found that the current level of knowledge about EVD among students and staff studied was low (median knowledge score $<50 \%)^{11}$. The study also found that science-based students had more knowledge than arts and social science-based students (median score $=46.2 \%, P<0.0001$ ). A similar study among University students in India found that $66.6 \%$ of the students had inadequate knowledge, $26.6 \%$ had moderate knowledge and $6.6 \%$ had adequate knowledge. Regarding the transmission of EVD, a study among college students in the USA found that $88 \%$ percent of them knew that the Ebola virus can be transmitted through "body fluids of an infected person," but $31 \%$ believed the virus can be transmitted through mosquitoes. ${ }^{12}$ A study in Lagos, Nigeria, found that $67 \%$ of the sampled health care workers had good knowledge of the Ebola virus disease, about 33\% had fair (average) knowledge while 6 percent had poor knowledge. ${ }^{13}$

\section{Aim of the study}

The aim of this study was to determine the knowledge, attitude and practice regarding Ebola virus disease among students of Nursing in the University of Calabar, Calabar, Nigeria.

\section{Objectives of the study}

The specific objectives of the study were:

1. To determine the knowledge of nursing students regarding EVD.

2. To determine the attitude of nursing students regarding EVD.

3. To determine the practice of nursing students regarding EVD.

\section{Methods \\ Study setting}

The University of Calabar is a second generation Nigerian University founded by the Federal Government of Nigeria in 1975. It is located in Calabar, the capital of Cross River State of Nigeria. The University currently has 15 faculties and three institutes. The Department of Nursing is in the Faculty of Allied Medical Sciences.

Study design: This was a descriptive, cross-sectional study.

Sample size determination: The sample size was calculated using the Leslie Kish formula:

$\mathrm{n}=\mathrm{Z}^{2} \mathrm{pq} / \mathrm{d}^{2}$

Where

$\mathrm{n}=$ minimum sample size 
$\mathrm{Z}=$ standard normal deviate which equals 1.96 at the $95 \%$ level of significance.

$\mathrm{P}=$ proportion of the desired attribute, which $=0.88 \mathrm{rep}-$ resenting the proportion of students who were found to have good knowledge of EVD in an earlier study ${ }^{12}$

$\mathrm{q}=1-\mathrm{p}$

$\mathrm{d}=$ degree of accuracy which is 0.05 .

Thus, the sample size was calculated to be 162. Making provision for $10 \%$ non-response rate, the sample size became $(162+16)=178$.
Sampling procedure: The Department of Nursing was purposively selected to participate in this study. Systematic random sampling was then used to select individual students to participants in the study.

\section{Results}

\section{Socio-demographic variables}

One hundred and sixty-seven (94.4\%) of the participants were females while $10(5.6 \%)$ were males. One hundred and seventy-two $(97.2 \%)$ respondents were Christians while $5(2.8 \%)$ belonged to other religions. Almost 50\% of respondents were in their third year of study while $13 \%$ were in their final (5th) year. (Table 1 )

\begin{tabular}{|c|c|c|}
\hline \multicolumn{3}{|c|}{ Table 1: socio-demographic characteristics } \\
\hline VARIABLE & FREQUENCY & PERCENT \\
\hline \multicolumn{3}{|l|}{ Sex } \\
\hline Male & 10 & 5.6 \\
\hline Female & 167 & 94.4 \\
\hline \multicolumn{3}{|l|}{ Age group } \\
\hline$<20$ & 85 & 48.0 \\
\hline $20-24$ & 43 & 24.3 \\
\hline $25-29$ & 23 & 13.0 \\
\hline $30-34$ & 23 & 13.0 \\
\hline$>34$ & 3 & 1.7 \\
\hline \multicolumn{3}{|l|}{ TRIBE } \\
\hline Efik/Ejagham/Bewkwarra & 36 & 20.3 \\
\hline Ibibio/Annang/Oron & 36 & 20.3 \\
\hline Igbo & 53 & 30.0 \\
\hline Others & 52 & 29.4 \\
\hline \multicolumn{3}{|l|}{ RELIGION } \\
\hline Christianity & 172 & 97.2 \\
\hline Islam & 5 & 2.8 \\
\hline \multicolumn{3}{|l|}{ MARITAL STATUS } \\
\hline Single & 160 & 90.4 \\
\hline Married & 17 & 9.6 \\
\hline \multicolumn{3}{|l|}{ YEAR OF STUDY } \\
\hline 3 & 88 & 49.7 \\
\hline 4 & 66 & 37.3 \\
\hline 5 & 23 & 13.0 \\
\hline
\end{tabular}

\section{Source of information regarding EVD}

Lecturers were the commonest source of information regarding EVD as reported by $84.5 \%$ of the respondents followed by television (76.3\%). Other sources of information regarding EVD were fellow students (37.3\%), family members $(9.6 \%)$, radio $(41.8 \%)$, Newspapers $(24.3 \%)$, internet $(36.2 \%)$, medical journals $(11.9 \%)$ and medical textbooks $(11.3 \%)$. (Table 2 ) 


\begin{tabular}{|l|l|l|}
\hline \multicolumn{3}{|c|}{ Table 2: Source of information regarding Ebola virus disease } \\
Source & Frequency* & Percentage* \\
\hline Fellow student & 66 & $37.3 \%$ \\
Family member & 17 & $9.6 \%$ \\
Radio & 74 & $41.8 \%$ \\
Television & 135 & $76.3 \%$ \\
Newspaper & 43 & $24.3 \%$ \\
Internet & 64 & $36.2 \%$ \\
Medical journal & 21 & $11.9 \%$ \\
Medical textbook & 20 & $11.3 \%$ \\
Lecturer & 150 & $84.5 \%$ \\
\hline
\end{tabular}

*Multiple responses allowed.

\section{Knowledge regarding EVD}

Over $66 \%$ of respondents indicated that the disease was named after a river in the Democratic Republic of Congo. Nearly $68 \%$ of respondents identified the monkey as the natural host of Ebola virus while chimpanzee was indicated by $27.7 \%$, gorillas $(35.6 \%)$, fruit bats $(39.5 \%)$ and swine $(11.9 \%)$. Regarding the mode of transmission of Ebola virus, $71.8 \%$ of respondents indicated direct contact with blood and other body fluids and secretions of infected persons while others indicated sexual intercourse $(29.4 \%)$, preparing and eating infected bush-meat $(58.8 \%)$, studying in the same room with someone who has not manifested symptoms of EVD (7.9\%) and shaking hands with someone who has not manifested symptoms of EVD $(7.9 \%)$. (Table 3)

Regarding the incubation period of EVD, $70.1 \%$ of respondents indicated that it is between 2 to 21 days while $17.5 \%$ indicated that it is 1 to 7 days. Forty-eight percent of respondents indicated that the first case of EVD in the world was reported in Liberia while $40.1 \%$ indicated that it was reported in the Democratic Republic of Con- go. Nearly $62 \%$ of respondents indicated that the first case of EVD in Nigeria was reported in July 2014 while about $12 \%$ indicated that it was in April, 2004. Majority of respondents, $92.1 \%$, indicated that internal and external bleeding are symptoms of EVD. Other symptoms indicated are fever $(67.8 \%)$, joint and muscle pains $(66.1 \%)$, headache $(71.8 \%)$ and nausea and vomiting (50.3\%) amongst others. (Table 3)

Regarding treatment of EVD, $71.8 \%$ of respondents indicated that there was no known cure yet, $15.8 \%$ reported that it could be treated with antibiotics like ampicillin while $12.4 \%$ indicated that only spiritual cure was recommended. Concerning prevention of EVD, 80.2\% of respondents indicated that patients should be treated in isolation centers, a similar proportion indicated regular hand washing and avoiding contact with blood and body fluids of EVD patients. Addressing myths and misconceptions was the preventive measure indicated by $15.8 \%$ of respondents while $12.4 \%$ indicated taking chemoprophylaxis each time one touched a suspicious patient. (Table 3) 


\begin{tabular}{|c|c|c|}
\hline KNOWLEDGE VARIABLE & FREQUENCY & PERCENT \\
\hline ORIGIN OF THE NAME EBOLA & & \\
\hline Named after Dr. Ebola, the first physician who diagnosed it. & 28 & $15.8 \%$ \\
\hline It was named after the village in Liberia & 33 & $18.6 \%$ \\
\hline It was named after a river in the Democratic Republic of Congo & 116 & $65.6 \%$ \\
\hline KNOWLEDGE OF NATURAL HOST OF EBOLA VIRUS & & \\
\hline Monkey & 120 & $67.8 \%$ \\
\hline Chimpanzee & 49 & $27.7 \%$ \\
\hline Gorillas & 63 & $35.6 \%$ \\
\hline Fruit bats & 70 & $39.5 \%$ \\
\hline Forest antelope & 75 & $42.4 \%$ \\
\hline Porcupine & 22 & $12.4 \%$ \\
\hline Swine & 21 & $11.9 \%$ \\
\hline KNOWLEDGE OF MODE OF TRANSMISSION OF EBOLA VIRUS & & \\
\hline Through fomites & 14 & $7.9 \%$ \\
\hline Contact with blood and body fluids/ secretions of infected persons & 127 & $71.8 \%$ \\
\hline Sexual intercourse & 52 & $29.4 \%$ \\
\hline Transfusion of infected blood & 64 & $36.2 \%$ \\
\hline Preparation and eating of infected bush-meat & 104 & $58.8 \%$ \\
\hline Shaking hands with someone who has not manifested symptoms & 14 & $7.9 \%$ \\
\hline $\begin{array}{l}\text { Studying in the same classroom with someone who has not } \\
\text { manifested symptoms of EVD. }\end{array}$ & 14 & $7.9 \%$ \\
\hline INCUBATION PERIOD OF EBOLA VIRUS DISEASE & & \\
\hline 1 t0 7 days & 31 & $17.5 \%$ \\
\hline 2 to 21 days & 124 & $70.1 \%$ \\
\hline WHICH COUNTRY REPORTED FIRST CASE IN THE WORLD? & & \\
\hline Liberia & 85 & $48.0 \%$ \\
\hline Democratic Republic of Congo & 71 & $40.1 \%$ \\
\hline WHEN WAS THE FIRST CASE REPORTED IN NIGERIA? & & \\
\hline January, 1999 & 11 & $6.2 \%$ \\
\hline April, 2004 & 21 & $11.9 \%$ \\
\hline July 2014 & 109 & $61.6 \%$ \\
\hline KNOWLEDGE OF SYMPTOMS OF EBOLA VIRUS DISEASE & & \\
\hline Fever & 120 & $67.8 \%$ \\
\hline Fatigue & 67 & $37.9 \%$ \\
\hline Joint and Muscle pain & 117 & $66.1 \%$ \\
\hline Headache & 127 & $71.8 \%$ \\
\hline Sore throat & 67 & $37.9 \%$ \\
\hline Nausea and vomiting & 89 & $50.3 \%$ \\
\hline Internal and external bleeding & 163 & $92.1 \%$ \\
\hline Redness of the eyes & 46 & $26.0 \%$ \\
\hline Rashes & 21 & $11.9 \%$ \\
\hline Polyuria & 21 & $11.9 \%$ \\
\hline
\end{tabular}

\section{Attitude regarding EVD}

One hundred and fifty-six (88.1\%) respondents indicated that the outbreak of EVD had put fear in them, 26.5\% indicated that the fear of EVD had dampened their interest and enthusiasm in the nursing profession while $30.5 \%$ indicated that they no longer felt comfortable traveling in public transport during the outbreak. Similarly, $12.4 \%$ indicated that they were no longer comfortable siting with fellow students in the classroom and $26.6 \%$ indicated that they were no longer comfortable going to the hospital ward. Over $72 \%$ of respondents indicated that they would encourage a roommate suspected of having 
EVD to go to the hospital, $24.3 \%$ indicated that they would pack out of the room and never return even after such roommate had been declared cured of EVD while $7.9 \%$ indicated that they would pack out and return to the room after the roommate had been declared cured of EVD. All $(100 \%)$ respondents indicated that they would continue to befriend someone who had been certified cured of EVD while $40.1 \%$ indicated that they would not eat food from a school food vendor who had EVD but had been certified cured. Similarly, $12.4 \%$ of respondents indicated that they would not take part as theatre nurse in the surgical operation of a patient who had been certified cured of EVD. (Table 4)

\begin{tabular}{|c|c|c|c|c|}
\hline \multicolumn{5}{|c|}{ Table 4. Attitude toward people with EVD } \\
\hline $\begin{array}{l}\text { WHAT WOULD YOU DO IF YOUR ROOM MATE IS SUSPECTED } \\
\text { TO HAVE EBOLA VIRUS DISEASE? }\end{array}$ & \multicolumn{2}{|c|}{ Frequency } & \multicolumn{2}{|c|}{ Percent } \\
\hline I would encourage her to go to the hospital & \multicolumn{2}{|l|}{128} & \multicolumn{2}{|c|}{$72.3 \%$} \\
\hline $\begin{array}{l}\text { I would pack out of the room and never return even after she } \\
\text { is declared cured. }\end{array}$ & \multicolumn{2}{|l|}{43} & \multicolumn{2}{|c|}{$24.3 \%$} \\
\hline $\begin{array}{l}\text { I would pack out of the room and return after she has been } \\
\text { declared cured }\end{array}$ & \multicolumn{2}{|l|}{14} & \multicolumn{2}{|c|}{$7.9 \%$} \\
\hline I would call the ebola hotline & \multicolumn{2}{|l|}{90} & \multicolumn{2}{|c|}{$50.8 \%$} \\
\hline $\begin{array}{l}\text { I would first buy medicine from the chemist for her and } \\
\text { encourage her to go to the hospital if the problem persists. }\end{array}$ & \multicolumn{2}{|l|}{22} & \multicolumn{2}{|c|}{$12.4 \%$} \\
\hline DICHOTOMOUS ATTITUDE RELATED VARIABLES & \multicolumn{2}{|c|}{ YES } & \multicolumn{2}{|c|}{ NO } \\
\hline $\begin{array}{l}\text { If your friend who had EVD has been certified cured of the } \\
\text { disease, would you be willing to continue to be his/ her } \\
\text { friend? }\end{array}$ & & $100 \%$ & 0 & $0 \%$ \\
\hline $\begin{array}{l}\text { If a food vendor in the school cafeteria is known to have had } \\
\text { EVD but is now certified cured, would you still eat the food } \\
\text { she prepares? }\end{array}$ & 92 & $52.0 \%$ & 71 & $40.1 \%$ \\
\hline $\begin{array}{l}\text { If you know that your patient had EVD but has been certified } \\
\text { cured, would you take part in his or her surgical operation as } \\
\text { the theatre nurse? }\end{array}$ & 155 & $87.6 \%$ & 22 & $12.4 \%$ \\
\hline $\begin{array}{l}\text { If you know that your patient who has come in labour had } \\
\text { EVD but has been certified cured, would you deliver her of her } \\
\text { baby? }\end{array}$ & 135 & $76.3 \%$ & 21 & $11.9 \%$ \\
\hline
\end{tabular}

\section{Practice regarding EVD}

All $(100 \%)$ respondents indicated that the outbreak of EVD caused them to increase the frequency with which they washed their hands. All respondents also indicated that the outbreak caused them to use personal protective equipment more often than before. Similarly, all respondents indicated that the outbreak caused them to carry their personal hand sanitizers. Only 22 (12.4\%) of respondents indicated that they would encourage their friends and family members to be volunteers in EVD vaccine trial just as $103(58.2 \%)$ respondents indicated their willingness to be volunteers in such a trial. Only $66(37.3 \%)$ of respondents had attended a workshop or training in EVD prevention. (Table 5) 
Table 5: practice regarding EVD prevention

PRACTICE VARIABLE

Did the outbreak of EVD cause you to increase the frequency with which you washed your hands?

Did the outbreak of EVD cause you to use Personal Protective

Equipment (e.g. hand gloves) more often than you used to?

Did the outbreak of EVD cause you to use hand sanitizer more

frequently?

Did you carry with you your own hand sanitizer during the

outbreak in Nigeria?

Did you write down or store in your phone any phone number of relevant agencies or organizations or persons to contact in case you suspected that you or someone you know had EVD?

Would you be willing to be a volunteer in EVD vaccine trial?

Would you also encourage your friends and family members to be volunteers in EVD vaccine trial?

In the event of an EVD outbreak in Nigeria, would you be willing to work in the EVD treatment/isolation center?

Have you attended any workshop/ training in EVD prevention?

Have you attended any workshop/ training in EVD case

management?

Do you think you still need further information/training regarding

EVD

RESPONSE

177

177

177

142

119

103

22

96

66

57

163

\begin{tabular}{l|ll|} 
YES & & NO \\
$100 \%$ & 0 & $0 \%$ \\
$100 \%$ & 0 & $0 \%$ \\
$100 \%$ & 0 & $0 \%$ \\
$80.2 \%$ & 21 & $11.9 \%$ \\
$67.2 \%$ & 58 & $32.8 \%$ \\
& & \\
$58.2 \%$ & 74 & $41.8 \%$ \\
$12.4 \%$ & 124 & $70.1 \%$ \\
$54.2 \%$ & 81 & $45.8 \%$ \\
$37.3 \%$ & 111 & $62.7 \%$ \\
$32.2 \%$ & 120 & $67.8 \%$ \\
$92.1 \%$ & 14 & $7.9 \%$ \\
\hline
\end{tabular}

\section{Test of association}

Association between students' year of study and some knowledge variables.

As shown in Table 6, although more students in year three, $64(36.16 \%)$ were likely to know the origin of the name Ebola than students in year four, 40(22.6\%) and year five, $12(6.7 \%)$, the difference was not statistically significant $\left(\mathrm{X}^{2}=4.543, \mathrm{df}=2, \mathrm{p}=0.1031\right)$. More students in year three, 66(44.59\%) were likely to know when the first case of Ebola Virus disease was diagnosed in Nigeria than students in year four, 31(20.95\%) and those in year 5 (12 (8.11\%). The difference was statistically significant $\left(\mathrm{X}^{2}=42.623, \mathrm{df}=2, \mathrm{p}=0.0000\right)$ 
Table 6: Association between students' year of study and some knowledge variables

\begin{tabular}{|c|c|c|c|c|c|c|}
\hline \multirow[t]{2}{*}{ Year of study } & \multicolumn{2}{|c|}{ Origin of the name Ebola } & \multirow[b]{2}{*}{ Total } & \multirow[b]{2}{*}{ Chi Square } & \multirow[b]{2}{*}{ Df } & \multirow[b]{2}{*}{ P-value } \\
\hline & Correct & Wrong & & & & \\
\hline 3 & $64(36.16 \%)$ & $24(13.56 \%)$ & $88(49.72 \%)$ & \multirow{4}{*}{4.543} & \multirow{4}{*}{2} & \multirow{4}{*}{0.1031} \\
\hline 4 & $40(22.60 \%)$ & $26(14.69 \%)$ & $66(37.29 \%)$ & & & \\
\hline 5 & $12(6.78 \%)$ & $11(6.21 \%)$ & $23(12.99 \%)$ & & & \\
\hline Total & $116(65.54 \%)$ & $61(34.46 \%)$ & $177(100.00 \%)$ & & & \\
\hline Year of study & \multicolumn{3}{|c|}{ Where first case of Ebola in the world was reported } & & & \\
\hline 3 & $28(15.82 \%)$ & $60(33.90 \%)$ & $88(49.72 \%)$ & \multirow{4}{*}{5.205} & \multirow{4}{*}{2} & \multirow{4}{*}{0.0740} \\
\hline 4 & $31(17.51 \%)$ & $35(19.77 \%)$ & $66(37.29 \%)$ & & & \\
\hline 5 & $12(6.78 \%)$ & $11(6.21 \%)$ & $23(12.99 \%)$ & & & \\
\hline Total & $71(40.11 \%)$ & $106(59.89 \%)$ & $177(100.00 \%)$ & & & \\
\hline Year of study & \multirow{2}{*}{\multicolumn{2}{|c|}{ When first Nigerian case was diagnosed }} & & \multirow{6}{*}{42.623} & \multirow{6}{*}{2} & \multirow{6}{*}{0.0000} \\
\hline & & & & & & \\
\hline 3 & $66(44.59 \%)$ & $0(0.00 \%)$ & $66(44.59 \%)$ & & & \\
\hline 4 & $31(20.95 \%)$ & $28(18.92 \%)$ & $59(39.87 \%)$ & & & \\
\hline 5 & $12(8.11 \%)$ & $11(7.43 \%)$ & $23(15.54 \%)$ & & & \\
\hline Total & $109(73.65 \%)$ & $39(26.35 \%)$ & $148(100.00 \%)$ & & & \\
\hline Year of study & \multirow{2}{*}{\multicolumn{2}{|c|}{ Incubation period of EVD }} & & \multirow{6}{*}{7.178} & \multirow{6}{*}{2} & \multirow{6}{*}{0.0276} \\
\hline & & & & & & \\
\hline 3 & $49(31.61 \%)$ & $17(10.97 \%)$ & $66(42.58 \%)$ & & & \\
\hline 4 & $52(33.55 \%)$ & $14(9.03 \%)$ & $66(42.58 \%)$ & & & \\
\hline 5 & $23(14.84 \%)$ & $0(0.00 \%)$ & $23(14.84 \%)$ & & & \\
\hline Total & $124(80.0 \%)$ & $31(20.0 \%)$ & $155(100.00 \%)$ & & & \\
\hline
\end{tabular}

\section{Discussion}

Television was the second most common source of information regarding EVD in this study. However, in a similar study among nursing students in Nellore, only $10 \%$ of respondents obtained the information from television whereas majority of them, $53.3 \%$ obtained information from newspapers. ${ }^{14}$ In this study, only $36.2 \%$ of students obtained information about EVD from the internet compared to $80.4 \%$ of undergraduate students in a study in Malaysia who indicated the internet as the first source of information on the disease. ${ }^{11}$ In a related study in the United States of America, only 11\% of respondents indicated "official government websites" as the source of information regarding EVD. ${ }^{12}$

Most of the respondents knew the correct mode of transmission of EVD, with as many as $71 \%$ correctly indicating one of the main modes of transmission. This is comparable to the findings of a similar study among students in Tehran, Iran where $74 \%$ of respondents knew the correct mode of transmission of the disease. ${ }^{15}$ About $12 \%$ of students in this study wrongly indicated that transmission of the disease can be prevented by taking chemoprophylaxis each time one touched a suspicious patient whereas in the Tehran study, as many as $46 \%$ of the respondents did not have any idea about how to prevent the transmission of the disease. ${ }^{15}$ In the study in the United States of America, 31\% of respondents indicated that EVD can be transmitted by mosquitoes. ${ }^{12}$ As high as $41.2 \%$ of respondents did not indicate preparation and eating of infected bushmeat as a means of transmission of EVD. This may be a form of denial. Bushmeat is a highly valued delicacy in Cross River State. Bushmeat eating restaurants, referred to locally as "bush meat joints" where bushmeat is prepared with local scent leaves and served with palm wine and plantain, are common both in the rural and urban areas of the state. Some people in the state say they would rather die than forego their favourite delicacy (bushmeat), irrespective of whatever disease may be related to it. 
Nearly $70 \%$ of respondents knew the correct incubation period of the disease. This proportion was comparable to the nearly $78 \%$ reported in a study among health care providers in Lagos state, Nigeria. ${ }^{13}$

Regarding the treatment of EVD, $71.8 \%$ of respondents in this study indicated that there is no known cure yet. This proportion was comparable to the $72.2 \%$ of respondents in Bayelsa state, Nigeria, who gave a similar response in a multi-site study in Nigeria. ${ }^{16}$ In the same multi-site study, only $41 \%$ of respondents from Kano gave the same response as the Bayelsa state respondents. ${ }^{16}$ Bayelsa and Cross River States are in Nigeria's Niger Delta region while Kano is in Northern Nigeria. A lot more social, economic and educational interactions, including sharing of knowledge and information, take place between residents of Bayelsa and Cross River States than with Kano state which is in the far North.

With respect to practice, this study found that only $45.8 \%$ of respondents indicated their unwillingness to work in the EVD treatment/isolation centre in the event of an EVD epidemic in Nigeria while $41.8 \%$ indicated their unwillingness to volunteer in EVD vaccine trial. These proportions are low when compared to the findings of the multi-site study in Kano, Bayelsa and Cross River States where $70 \%$ of health care workers in Calabar indicated their unwillingness to work in units caring for EVD patients. ${ }^{16}$ The difference may be explained by the fact that the respondents in our study are still in training to become health care providers (nurses) and may not be as afraid of contacting infectious diseases from patients as those who are already in practice which were the respondents in the multi-site study. Those who are already in practice may have seen practical cases of transmission of infections from patients to their health care providers while the students of nursing may only be hearing and reading about it theoretically.

Interestingly, only $7.9 \%$ of respondents indicated that studying in the same classroom with someone who has not manifested symptoms of EVD can lead to contacting the virus. Similarly, $7.9 \%$ of respondents indicated that if a roommate was suspected to have EVD, they would pack out of the room and return after they had been declared cured. These low proportions may be an indication that stigma and discrimination against people suspected of having EVD would be low. This also reflects in the finding that all the respondents $(100 \%)$ indicated that if a friend who had EVD became certified cured, they would be willing to continue with the friendship. It also reflects in patient care as $76.3 \%$ indicated that if they knew that their patient who had come in labour had EVD but had been certified cured, they would deliver her of her baby. Similarly, $87.6 \%$ indicated their willingness to be part of the surgical operation of their patient who had been certified cured of EVD whereas in the study among healthcare providers in Lagos, Nigeria, only $43 \%$ of respondents indicated their willingness to join a volunteer corps of visiting physicians in providing health care to Ebola virus disease patients. ${ }^{13}$ Surprisingly, the good attitude of respondents in this study towards people cured of EVD did not reflect in their relationship with the school food vendor as only $52 \%$ of them indicated their willingness to patronize them if they were certified cured.

Nearly $16 \%$ of respondents indicated that one of the ways of preventing EVD is by addressing myths and misconceptions. This is very important because myths and misconceptions regarding health-related phenomena are very common in Nigeria. The belief in witches and wizards and evil spirits as the cause of virtually every health-related event influences the health-seeking behavior of the people. During the EVD outbreak in Nigeria, several myths and misconceptions circulated in the social media. ${ }^{17,18}$. This led to such unwholesome practices as having salt water bath and drinking salt water, eating of bitter kola, drinking one's urine, walking naked around the compound at midnight, consultation of spiritual medium, recitation of incantations and sacrificing to the gods, as the means of preventing and curing the disease. Myths and misconceptions regarding Ebola have also been reported by other researchers and reports. ${ }^{19,20,21}$ The WHO had indicated that bursting myths was crucial to stopping the transmission of EVD in Guinea. ${ }^{22}$

Only $32.2 \%$ of respondents in this study had attended workshop/training in EVD case management while 
$37.3 \%$ had attended workshop/training on EVD prevention. These proportions are high compared to the $16.6 \%$ of respondents in a study among health care providers in Lagos, Nigeria, who had received training on how to identify suspected EVD patients. ${ }^{13}$

\section{Conclusion}

Majority of the students were knowledgeable about EVD. Although majority of them had favorable attitude towards EVD patients, there was evidence of stigmatizing and discriminatory attitude that need to be addressed. This is necessary in order to ensure that these students graduate to become providers of unbiased health care to all categories of patients.

\section{Declaration of conflict of interest}

The authors have no conflict of interest to declare.

\section{References}

1. WHO: Ebola Virus Disease http://www.who.int/mediacentre/factsheets/fs103/en/

2. Ebola haemorrhagic fever in Sudan, 1976. Report of a WHO/ International Study Team. Bull World Health Org 1978;56:247-70.

3. World Health Organization. Ebola virus disease, West Africa update. Disease Outbreak News, April 17, 2014. <www.who.int/csr/don/2014_04_17_ebola/en/>

4. Centers for Disease Control [CDC]. Ebola Virus Disease. Available: http://www.cdc.gov/vhf/ebola/ index. html. Accessed10thDecember2014

5. Beeching N J, Fenech M, Houlihan CF. Ebola Virus Disease. BMJ 2014;349 :g7348doi:10.1136/bmj. g7348PMID:25497512

6. Feldmann H, Geisbert TW. Ebola haemorrhagic fever. Lancet2011; 377(9768):849-62.doi:10.1016/ S01406736(10)60667-8. PubMed

7. European Centre for Disease Prevention and Control[ECDC]. Outbreak of Ebolavirus disease in West Africa. Thirdupdate, 1August2014. Stockholm: ECDC;2014.

8. Ndambi R, Akamituna P, Bonnet M, Tukadila A, Muyembe-Tamfum J, Colebunders RL. Epidemiologic and clinical aspects of the Ebola virus epidemic in Mosango Democratic Republic of the Congo,1995. The Journal of Infectious Diseases 1999; 179:S8-S10PMID:9988156. 9. World Health Organization (WHO), Ebola Situation Report, 2014, http://apps.who.int/ebola/en/status-out- break/situationreports / ebola-situation-report-31-december-2014.

10. WHO declares end of Ebola outbreak in Nigeria http:/ /www.who.int/mediacentre/news / statements/2014/nigeria-ends-ebola/en/).

11. Abubakar U, Sulaiman SA. Knowledge of Ebola virus disease: An evaluation of university students and staff regarding the current Ebola issue around the globe. Arch Pharma Pract 2015; 6:85-92 PubMed

12. Koralek T, Assessing the level of knowledge, attitude and beliefs about Ebola Virus Disease among college students. Am J Infect Control. 2015 Oct 1;43(10):11435. doi: 10.1016/j.ajic.2015.06.012. Epub 2015 Jul 29. PubMed

13. Oladimeji AM, Gidado S, Nguku P, Nwangwu IG, Patil ND, Oladosu F, Roberts AA, Waziri NE, Shuaib F, Oguntimehin O, Musa E, Nasidi A, Adewuyi P, Olayinka A, Odubanjo O; N-FELTP Residents, Poggensee G. Ebola virus disease - gaps in knowledge and practice among healthcare workers in Lagos, August 2014.Trop Med Int Health. 2015 Sep;20(9):1162-1170. doi: 10.1111/ tmi.12528.

14. Ramanjamma K, Indira S. Knowledge regarding Ebola virus disease and prevention among nursing students in selected colleges at Nellore. RNNJ. Narayana Nursing Journal 2015; 4(4): 38-41

15. Holakouie-Naieni K, Ahmadvand A, Raza O, Assan A, Elduma AH, Jammeh A, Kamali AS, Kareem AA, Muhammad FM, Sabahat H, Abdullahi KO, Saeed RA, Saeed SN. Assessing the Knowledge, Attitudes, and Practices of Students Regarding Ebola Virus Disease Outbreak. Iran J Public Health. 2015 Dec;44(12):1670-6.

16. Iliyasu G, Ogoina D, Otu AA, Dayyab FM, Ebenso B, Otokpa D, Rotifa S, Olomo WT, Habib AG. A Multi-Site Knowledge Attitude and Practice Survey of Ebola Virus Disease in Nigeria. PLoS One. 2015 Aug 28;10(8):e0135955. doi: 10.1371/journal.pone.0135955. eCollection 2015.

17. All Africa-Nigeria: Hot Water, Salt Not an Ebola Cure (2014). Available: http://allafrica.com/view/ group/main/main/id/00031909.html

18. Oyeyemi SO, Gabarron E, Wynn R. Ebola, Twitter, and misinformation: a dangerous combination? BMJ 2014; 349. http://dx.doi.org/10.1136/bmj.g6178. PubMed 
19. Catholic Relief Services. Study on Public Knowledge, Attitudes, and Practices Relating to Ebola Virus Disease (EVD) Prevention and Medical Care in Sierra Leone 2014.

20. Adongo PB, Tabong PT-N, Asampong E, Ansong J, Robalo M, Adanu RM. Beyond Knowledge and Awareness: Addressing Misconceptions in Ghana's Preparation towards an Outbreak of Ebola Virus Disease. PLoS One 2016;11:e0149627. pmid:26889683

21. Kpanake L, Gossou K, Sorum PC, Mullet E. Misconceptions about Ebola virus disease among lay people in Guinea: Lessons for community education. J Public Health Policy 2016; 37:160-72. pmid:26865320

22. WHO. Busting the myths about Ebola is crucial to stop the transmission of the disease in Guinea. http:// www.who.int/features/2014/ebola-myths/en/ 